\title{
FUSION OF LASER AND IMAGE SENSORY DATA FOR 3-D MODELING OF THE FREE NAVIGATION SPACE
}

\author{
M.Maas, A.Moghaddamzadeh and N.Bourbakis \\ Binghamton University \\ T.J.Watson School \\ Dept. EE, AAAI Lab \\ Binghamton, NY 13902
}

\begin{abstract}
In this paper a fusion technique is presented for the 3-D modeling of the free navigation space. The fusion technique could be used by an autonomous robot to model and acquire the navigation space and for the extraction of the 3-D map from the environment. The fusion technique is based on the appropriate synthesis of two different sensory data generated by a vision camera and a laser scanner.
\end{abstract}

KEYWORDS: Fusion, Image Segmentation, Laser Scanning, 3-D images

This work is a part of an FR grant 1992-93

\section{INTRODUCTION}

Humans and animals are born with multiple senses that allow them to develop a very clear picture of our world. Thus, in the design of autonomous robots, or robust vision systems, the cooperation of the multiple sensors is needed to enhance the system's chances of success in a complex environment. Of particular importance is how the robot formulates a 3-D image of the environment. We intend on using the fusion of a color segmented picture and a laser range finder to develop a working model of the space.

The idea of fusing two sensory signals to formulate a 3-D image is by far not new. Extensive work has been done in this field of machine vision. We encourage the reader to further enhance their background by looking at [1] and [2].

Several very interesting approaches have been done in the field of fusing intensity and range data. Of particular interest is the work done by J.K. Aggarwal et. al. [3].

In particular in [3], a method of fusion between range data and intensity is presented. One major goal of this approach was to minimize the extensive amount of time required in sensing the range data. Using potential points of interest from the intensity data as directors for the range sensor, the range sensor senses those points of interest and then the range data and intensity data are combined to form the graph.

Another method is discussed in [4]. This method uses the most dominant sensor at one time (range or intensity) for seed segmentation. This segmented area then again uses the data from the range and intensity for region merging. The authors used this method to minimize the lighting difficulties and color variance (intensity image segmentation), and to minimize the difficulties range image segmentation has with sloped surfaces.

Other related work is available in [5],[6], and [7].

It is interesting to be mentioned that the laser scanner has very high resolution (similar to a digital image) very close to the scene surface. This means that in longer distances, such as $1 \mathrm{~m}$ or longer than that, the resolution changesdrastically. For instance, in a distance of $1 \mathrm{~m}$, the size of the laser spot has diameter of $1 \mathrm{~cm}$ approximately. Thus, how the image 3-D model will be developed under these conditions.

We propose a methodology, which hopefully will correct some of these problems, such as long distance 3D image modeling and color difficulties, by using the intensity data as the director for the laser. More specifically, we use a fuzzy image segmentation technique [9] for the sufficient separation of the image regions with different color. In addition, we generate an image with a low resolution equivalent to the laser one, thus the fusion of these sensory data to be possible and the final 3-D image to contain less "noise" on the scanned surfaces.

This paper is organized into five sections. Section 2 discusses the laser range and some of the existing problems. Section 3 presents briefly the fuzzy image segmentation technique for the separation of the regions. Section 4 describes the fusion methodology of these two different sensory data. The last section summarizes the overall presentation. 


\section{LASER RANGE DATA ACQUISITION}

Ideally we would like to scan the picture area with a laser scanner. This would provide us with a range map that could be "fused " with our intensity segmented map. However the inhibitive high cost of laser scanners is not within our university's budget.

That leaves us with two options; we can attempt to build our own laser scanner or we can purchase a point by point laser range detector that can be manipulated in a xy plane. Due to the time constraints of this paper we will assume that we have the funds to purchase a point by point detector. It is also worth noting that it would probably take us a considerable amount of time to build the complex circuitry required for a laser scanner. Our objective is to develop a new fusion technique.

One company that has a variety of laser distance meters is RIEGL[8]. Features included in these detectors are RS233 interface (as long as measure times are $>\mathbf{5 0} \mathrm{ms}$ ), immunity to electro-magnetic and acoustic interference, high speed and accuracy, and a starting beam width of $\approx 1 \mathrm{~cm}$ with a divergence of $3.2 \mathrm{mrad}$. Since our robot is designed primarily for enclosed hazardous environments the laser sensor range does not need to be exceptionally long. The meter fulfilling our needs the best is the LD90-210-CX/C6. It's sensing range is from 1 meter to $\mathbf{1 5}$ meters. In addition [8] also has a biaxial rotary mount (BD 90). The mount enables us to selectively pick the intensity segmented areas.

Theoretically a beam width the size of a pixel would be ideal. This would permit a one to one fusion with the pixels form the intensity segmentation. However technology has not yet reached this minute beam width. The authors of this paper are aware of very small diameter beam widths but these lasers are not yet available.

After the separation of the intensity scan into specific colors the area of each color segment is determined and put in xy coordinates. Once an area is defined the bidirectional mount is manipulated so that the laser range meter can determine the range of the area in question. When the scanning of the area is complete another color area is scanned until the entire intensity frame has been scanned. The intensity map and the range map are then fused to form our first attempt at the 3-D image.

One of the critical features of the laser scanner is the non-uniform scanning of the scene. More specifically, since the mechanical XY movement mechanism used by the laser scanner is imperfect, there is the possibility that the laser beam to overlap two consecutive scanned point, or to be far away from each other, as shown in figure 1. A solution to this particular problem is the scanning of the same scene area twice, and the averaging of two distance values by reducing the possible error.
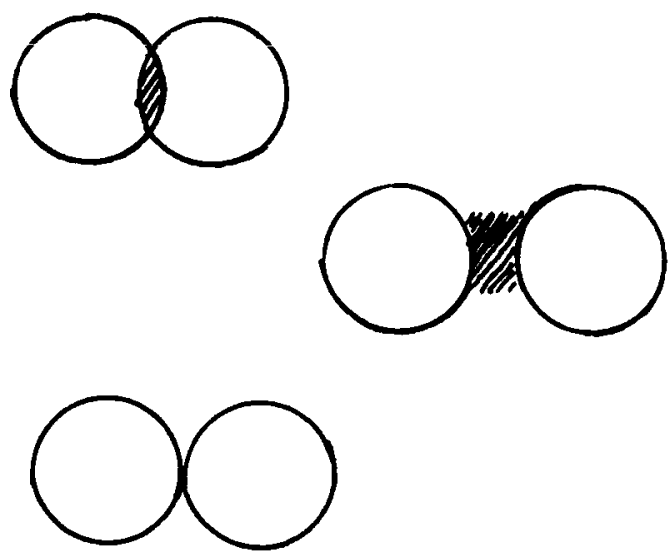

Figure 1: The imperfect scanning

\section{FUZZY IMAGE SEGMENTATION}

Segmentation and edge detection in color images are not deeply investigated in the literature. Some methods separate the three color components and work on each independently. Three different histograms are drawn and after segmentation or edge detection, the results are combined to form segmented or edge detected color images. The results using these techniques are false because of three reasons: one, segmentation or edge detection based on color alone is not complete. The relative position of the pixels and their color in their neighborhood are important as well. Two, in real images there is no sharp valley in histograms. Three, once one pixel color is mapped to three locations on three histograms, pixel color information is scattered. And once this is done for thousands of pixels, all available information is scattered and mixed. After thresholding doing the reverse and putting the three components back together is not an easy job. This technique is usually used for images having a limited number of segments or clusters.

The technique described in this section is applicable to both color and gray level images having an unlimited number of clusters. A cluster is defined as a collection of touching pixels which have almost the same color or the change in color is gradual. Since an unlimited number of clusters could be detected from an image, displaying each cluster in a different image would not be practical. What we have done is displaying all clusters in one image. Each cluster is shown using a color that reflects the main color of the cluster.

Before segmentation can begin a rough edge detection must be performed. The used edge detection technique is not described here but it is sensitive enough to detect all sorts of edges (crisp, fužy, thin, and week). A histogram table is also generated and sorted according to the number of pixels. The first three entries of the table for a particular image are shown below: 
212723892

212623640

81228591

The numbers represent red, green, blue, and the number of pixels in the image having that particular color vector, respectively. Segmentation is performed in the following steps: 1- find big clusters, 2- expand clusters, 3- find medium size clusters, 4- expand clusters, 5-find small clusters, 6 - fill in the blanks.

Big clusters are defined as those having a minimum of 150 pixel members. To find big clusters instead of looking for valleys in the histogram we look for peaks. The first peak is the first entry in the histogram table. Therefore, the seed color is known and the seed location must be found. The image is scanned for that particular color vector and once found it is test-grown and pixels marked until a detected edge is reached and the size of the test-grown cluster is noted. This is done for all unmarked pixels having the seed color. The seed location that uses the most of the seed color pixels and is big is chosen as the seed location and its color as the cluster color. The seed is then grown. While the seed is growing and pixels are added to the cluster, each pixel is marked and its entry in the histogram table decrement. Meaning, that particular pixel is not available any more and does not contribute to the histogram table. When the seed is all grown, the histogram table is resorted and the first entry, now, is the second peak of the histogram. This growing process is repeated until there are no more big clusters left.

Since the edge detection algorithm used is very sensitive to variance, the detected edges are thick especially in the case of fuzzy edges. Therefore, big clusters must be expanded using fuzzy logic to fill out that gap before smaller clusters are found. Big cluster expansion is performed in two steps: In the first step each cluster is expanded (surrounding pixels added to the cluster) based on two rules:

1. The absolute variance (difference between the pixel's color and the seed/cluster's color) is low. and

2. The relative variance (difference between the next and the previous pixel's color) is low. This is used to include gradual changing shade areas as well.

In the second step each cluster is expanded based on the following rule:

For each pixel the absolute variance with the surrounding clusters and the distance (in number of pixels) with that cluster is evaluated and their product recorded (degree of farness). If the lowest product value (which is for the closest cluster in terms of color and distance) is lower than a threshold, the pixel is added to the cluster.

The following equation is used to calculate variance between pixel 1 and 2 .

$$
\text { Variance }_{1,2}=\left(R_{2}-R_{1}\right)^{2}+\left(G_{2}-G_{1}\right)^{2}+\left(B_{2}-B_{1}\right)^{2}
$$

where $R, G$, and $B$ are the three color components. Variance membership function is shown below:

$$
\mu_{V_{1,2}}=\left\{\begin{array}{lr}
0 & \text { if Variance } \leq a_{1} \\
1 & \text { if Variance }{ }_{1,2} \geq a_{2} \\
\frac{\text { Variance }_{1,2}-a_{1}}{a_{2}-a_{1}} & \text { else }
\end{array}\right.
$$

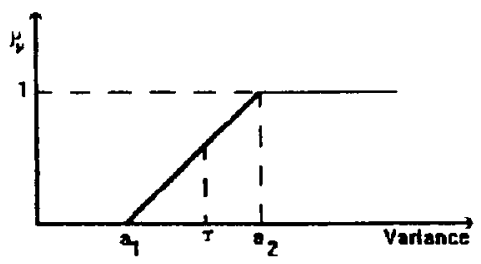

Averaging is used in anding the two fuzzy membership functions.

Medium size clusters are found the same way big ones were but this time they are grown based on the first step of expansion rules (minimum size 30 pixels total). They are then expanded based on the second step of expansion rules.

To find small clusters, for each undecided pixel in the image the degree of farness with the nearby clusters is evaluated.

If the lowest value is higher than a threshold, use the pixel as a seed and test-grow it based on the first step of expansion rule. If the test-grown cluster has at least 6 pixel members and has a big contrast with the closest cluster (its existence is of importance), grow it, otherwise leave it undecided. To fill in the blank for all undecided pixels the closest cluster (in distance and color) is found and the pixel is added to that cluster. Figure 2 shows a colored image and its segmentation. Figure 3 shows a grey image followed by its segmented one.

\section{THE FUSION METHODOLOGY}

\subsection{Methodology}

The fusion methodology presented here is based on the synthesis of different sensory data generated by a vision system and a laser scanner. In particular, the camera receives an image from the environment. The digital form of the image $P(i, j), 1<i, j<n$, where " $n$ " represents the size of the image matrix, is stored in the main memory. The digital image represents a 2-D array of gray level values or color values $(g v(i, j))$ for the image pixels. At the time that the image is grabbed by the camera, the laser scanner "scans" the same area of the 


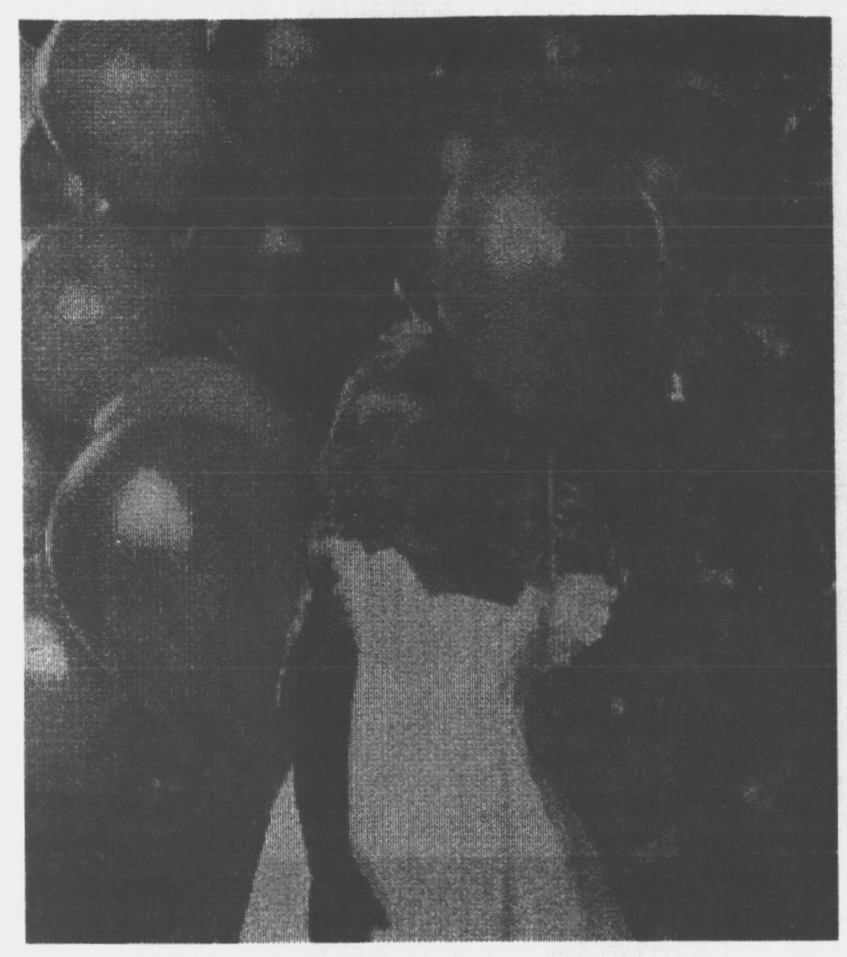

(a)

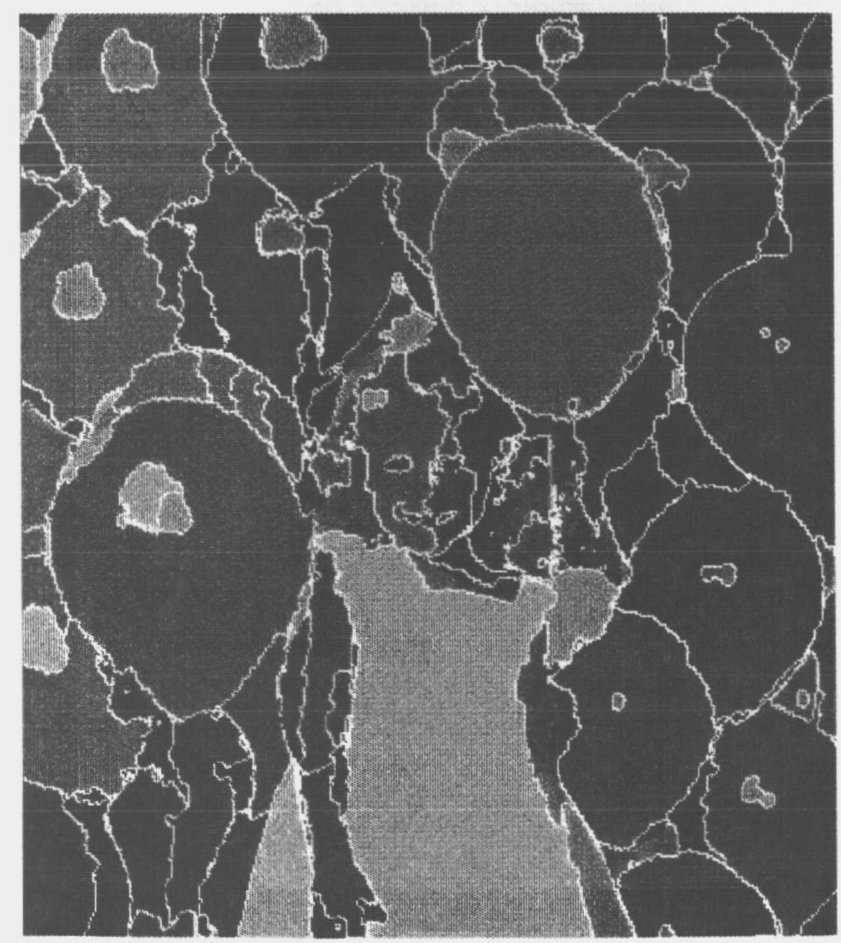

(b)

Figure 2: a) A colored image

b) Its segmentation

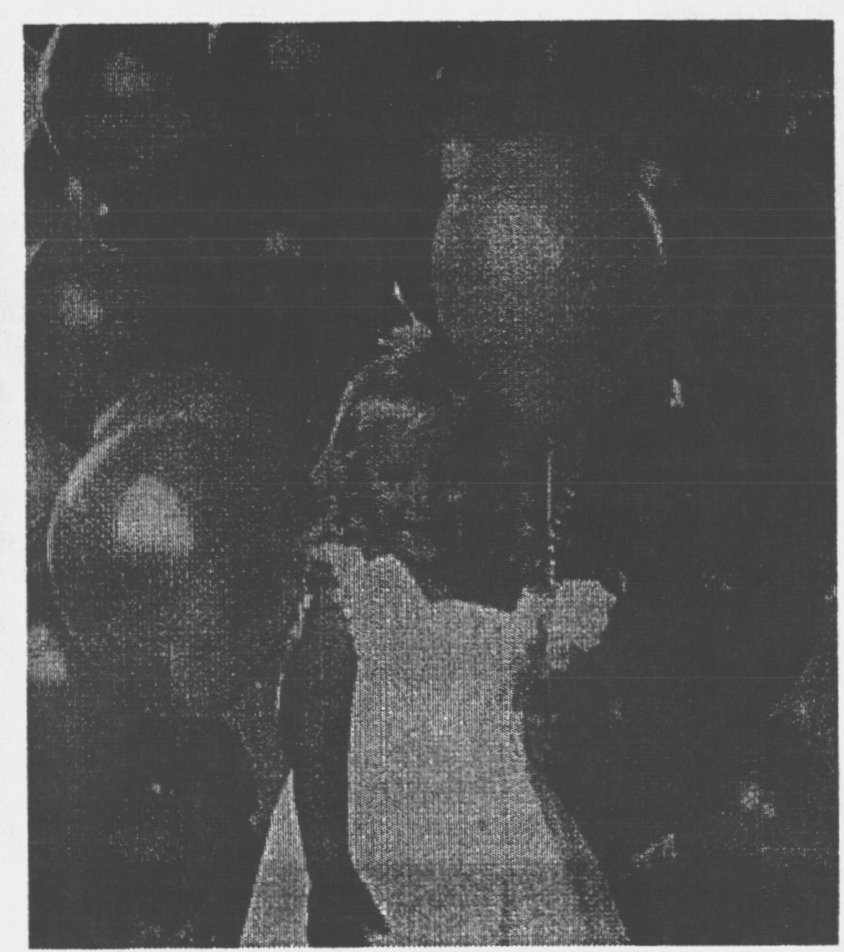

(a)

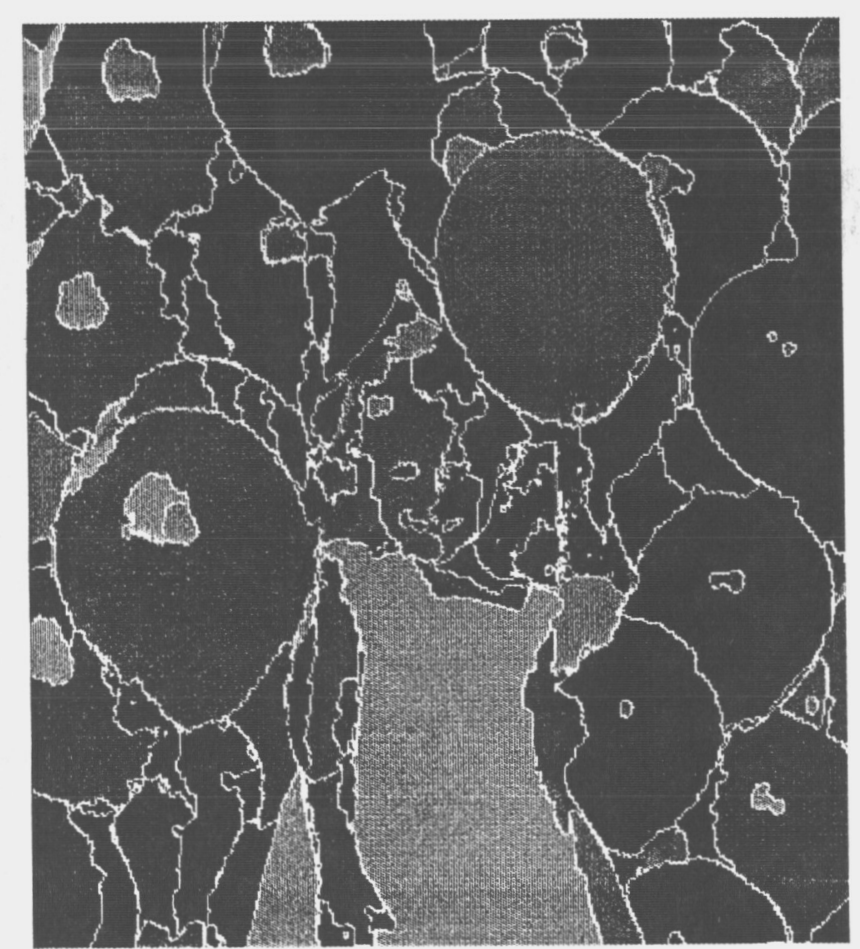

(b)

Figure 3: a) A grey level image

b) Its segmentation 
environment by producing an 2-D array $D(k, m)$. The values $d(k, m)$ of the $D$ array represent the distances of the laser scanner from the surface of the current navigation space at the particular area from which the image was grabbed. The $D$ array is also stored in the memory. From this point, the fusion technique takes place. More specifically, a fuzzy segmentation technique is applied on the image values to define the regions with "color" similarities. An edge detection technique is also applied on the same image to define and separate the boudaries of the image regions [9]. Since the resolution of the $\mathbf{P}$ image-matrix is higher to the $\mathbf{D}$ distance-matrix, the resolution ratio $(R(F)=[R(P) / R(D)])$ is calculated. This $R(F)$ ratio will guide the image pyramid generation technique for the selection of the pyramidal level of the image (PL) with the closest resolution to the D matrix, figure 4.

At this point, the fusion matrix $F$ is generated by combining the coordinates of these two matrices PL and D respectively. More specifically, the elements of the $F$ matrix have four values: the $x^{\prime}$ and $y^{\prime}$ coordinates, the d distance of these $x^{\prime} y^{\prime}$ coordinates, and the gv grey level value which cooresponds to $x^{\prime} y^{\prime} ; f\left(x^{\prime}, y^{\prime}, d, g v\right)$. The 3-D image model (F3) for the original image will be generated by the $\mathrm{F}$ matrix with the expansion of each f(x'y',d,gv) element by $R(F)$ pixels. More specifically, $f\left(x^{\prime}, y^{\prime}, d, g v\right) \rightarrow S\{p\}$

where $S$ represents the set of $4^{q}$ neighbor pixels, so that $R(F) \leqslant 4^{9}$. Thus, $4^{9}$ neighbor pixels of the 3-D image model will share the same distance d; $p(i+w, j+z, d, g v)$, where $w, z e\left\{1,2, \ldots 2^{q}\right\}$.

At this point, the colored image regions separated by the segmentation method will be used for "smoothing" the possible unevenness of the 3-D model. More specifically, there will be cases where the pixels, which belong to different colored regions, share the same distance $d$. In this case, the distance $d(R c)$ which represents the majority of the pixels in the region $\mathrm{Rc}$, with color "c", will be used to redefine the distance of those pixels, which share a distance different than $\mathrm{d}(\mathrm{Rc})$.

\subsection{Illustrative Example}

In this subsection we present graphically the fusion methodology by using a simple grey level image, figure 5 , the reduced image using the pyramidal process, figure 6, a generated distance matrix with equivalent resolution, figure 7 , the fused 3 -D image at the reduced size, figure 8 , and the 3-D model of the original image, figure 9.

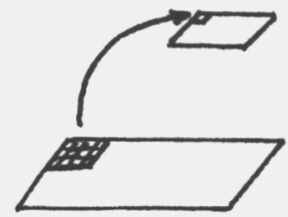

Figure 4: The pyramidal reduction of an image

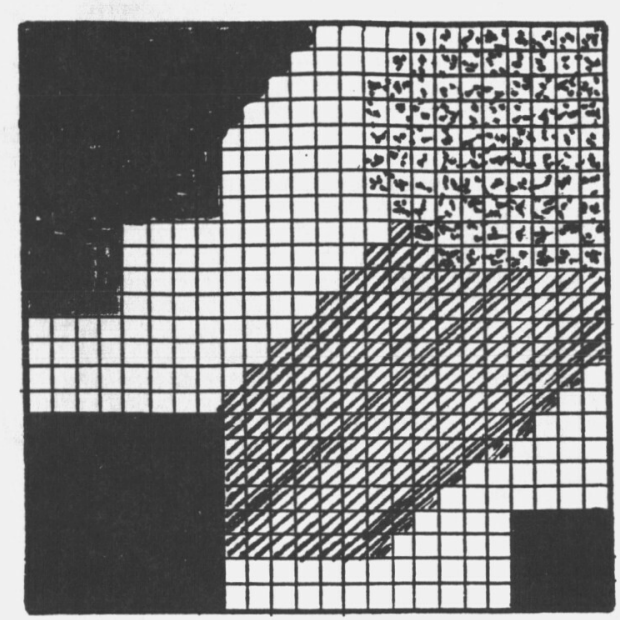

Figure 5: A grey level image

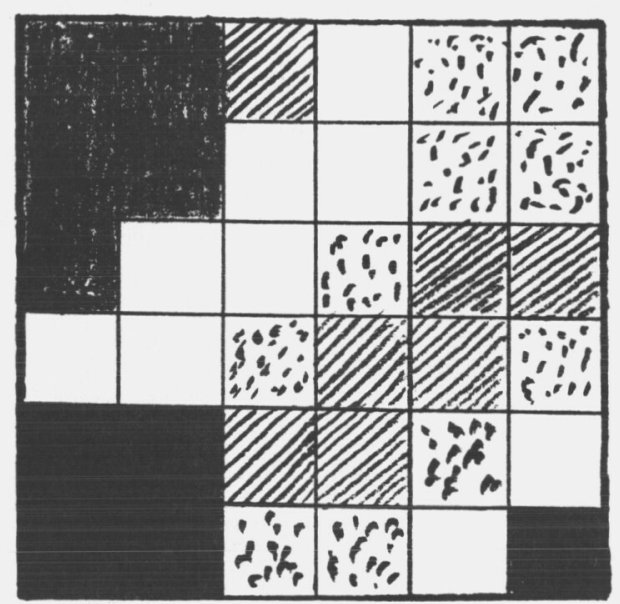

Figure 6: The reduced image using pyramidal procedure.

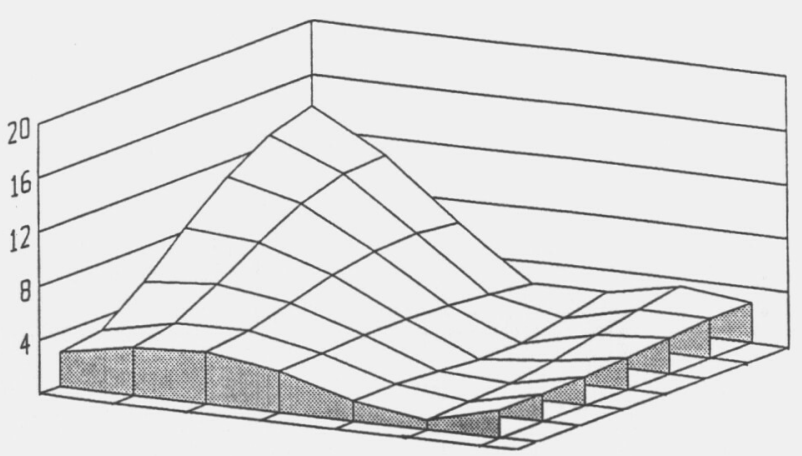

Figure 7: The distance matrix (in a 3-D form) 


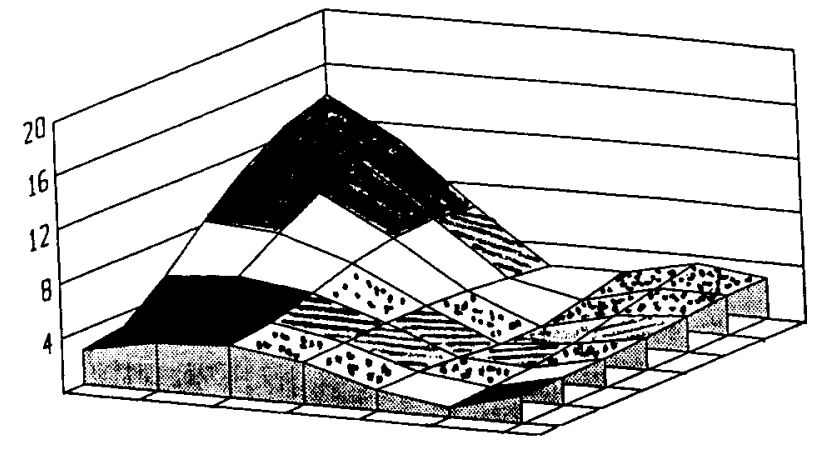

Figure 8: The fused image at the reduced size

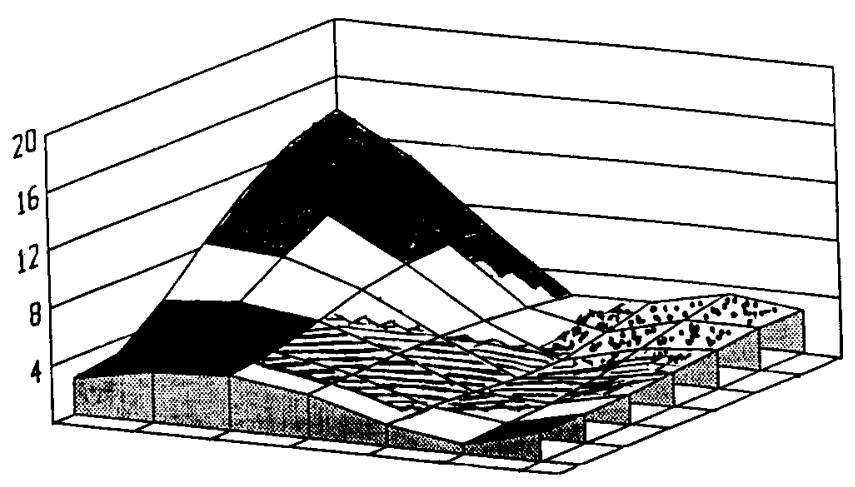

Figure 9: The 3-D model of the original image

\section{REFERENCES}

[1] J.K. Hackett and M. Shah, "Multi-Sensor Fusion: A Perspective," IEEE International Conference on Robotics and Automation, pp. 13241330, 1990.

[2] J.K. Aggarwal and A. Mitiche, "Multiple Sensor Integration/Fusion Through Image Processing: A Review," Optical Engineering, 25:380-6, March 1986.

[3] M.J. Magee, B.A. Boyter, C.H. Chien, and J.K. Aggarwal,"Experiments in Intensity Guided Range Sensing Recognition of Three-Dimensional Objects," IEEE Trans. Pattern Anal Machine Intell, vol. PAM1-7, pp. 629-637, Nov. 1985.

[4] J.K. Hackett and M. Shah, "Segmentation Using Intensity and Range Data," Optical Engineering, 28:667-674, June 1989.

[5] B. Gill, A. Mitiche, and J.K. Aggarwal, " Experiments in combining intensity and range edge maps," Comp. Graph. Image Pro. 21, 395-411 (1983).

[6] M. Hebert, "Outdoor scene analysis using range data," IEEE Conf. on Robotics and Automation 3, 1426-1432 (1986).

[7] R.O. Duda, D. Nitzan, and P. Barrett, "Use of range and reflectance data to find planar surface regions," IEEE Trans. Pattern Anal. Machine Intell, PAMI-1, 259-271, 1979.

[8] RIEGL LASER MEASUREMENT SYSTEMS, RIEGL USA INC., 4419 Parkbreeze Court, Orlando, Florida 32808

[9] A.Moghaddanzadeh and N.Bourbakis," A fuzzy image segmentation-edge detection technique", TR1993, sub.

\section{CONCLUSIONS}

In this paper a fusion methodology has been presented. This methodology combines two different type of sensory data for the generation of a 3-D image model. The sensory data used by this method were the distances produced by a laser scanner and the pixels average intensities (or color) of a 2-D digital image grabbed by a vision camera. The problem of the different resolutions for these two sensory data was solved by the reduction of the image resolution, the fusion of the different data and the reconstruction of the original image by using a fuzzy image segmentation technique. Since the laser scanner was available for this experimental work, only feasibility simulated results were presented. 